Pure and Applied Mathematics Quarterly

Volume 5, Number 2

(Special Issue: In honor of

Friedrich Hirzebruch, Part 1 of 2)

$701-727,2009$

\title{
A Composition Formula for Manifold Structures
}

Andrew Ranicki

For Prof. F. Hirzebruch

\begin{abstract}
The structure set $\mathcal{S}^{T O P}(M)$ of an $n$-dimensional topological manifold $M$ for $n \geqslant 5$ has a homotopy invariant functorial abelian group structure, by the algebraic version of the Browder-Novikov-Sullivan-Wall surgery theory. An element $(N, f) \in \mathcal{S}^{T O P}(M)$ is an equivalence class of $n$-dimensional manifolds $N$ with a homotopy equivalence $f: N \rightarrow M$. The composition formula is that $(P, f g)=(N, f)+f_{*}(P, g) \in \mathcal{S}^{T O P}(M)$ for homotopy equivalences $g: P \rightarrow N, f: N \rightarrow M$. The formula is required for a paper of Kreck and Lück.
\end{abstract}

Keywords: Topological manifold, structure set, surgery theory

\section{INTRODUCTION}

The structure set $\mathcal{S}^{T O P}(M)$ of an $n$-dimensional topological manifold $M$ is the pointed set of equivalence classes of pairs $(N, f)$ with $N$ an $n$-dimensional manifold and $f: N \rightarrow M$ a homotopy equivalence, with $\left(N_{1}, f_{1}\right)=\left(N_{2}, f_{2}\right) \in$ $\mathcal{S}^{T O P}(M)$ if and only if $\left(f_{1}\right)^{-1} f_{2}: N_{2} \rightarrow N_{1}$ is homotopic to a homeomorphism, and $(M, 1) \in \mathcal{S}^{T O P}(M)$ the base point. The equivalence class $(N, f) \in \mathcal{S}^{T O P}(M)$ is called the structure invariant of $(N, f)$. For $n \geqslant 5 \mathcal{S}^{T O P}(M)$ is determined by 
the fundamental exact sequence of pointed sets of the Browder-Novikov-SullivanWall surgery theory

$$
\cdots \longrightarrow L_{n+1}\left(\mathbb{Z}\left[\pi_{1}(M)\right]\right) \longrightarrow \mathcal{S}^{T O P}(M) \stackrel{\eta}{\longrightarrow} \mathcal{T}^{T O P}(M) \stackrel{\theta}{\longrightarrow} L_{n}\left(\mathbb{Z}\left[\pi_{1}(M)\right]\right)
$$

with $\mathcal{T}^{T O P}(M)=[M, G / T O P]$ the normal bordism set, $G / T O P$ the classifying space for fibre homotopy trivialized topological bundles, $\eta$ sending a structure invariant to its bordism class when regarded as a normal map, and $\theta$ the surgery obstruction function.

This paper will only consider homotopy equivalences of manifolds which are simple, i.e. have zero Whitehead torsion, in order to take advantage of the $s$-cobordism theorem. However, all the results have analogues for homotopy equivalences which are not necessarily simple.

The algebraic surgery sequence of abelian groups

$$
\cdots \rightarrow L_{n+1}\left(\mathbb{Z}\left[\pi_{1}(M)\right]\right) \rightarrow \mathcal{S}_{n+1}(M) \rightarrow H_{n}\left(M ; \mathbb{L}_{\bullet}\right) \stackrel{A}{\rightarrow} L_{n}\left(\mathbb{Z}\left[\pi_{1}(M)\right]\right) \rightarrow \cdots
$$

was constructed in Ranicki $[17, \S 16]$ for any space $M$, with $\mathbb{L}_{\bullet}$ the 1-connective quadratic $L$-theory spectrum of $\mathbb{Z}$ such that $\mathbb{L}_{0} \simeq G / T O P$, and with $A$ the assembly map. Each of the terms in the sequence is a homotopy invariant functor of $M$, and the morphisms are natural. In particular, a map of spaces $f: N \rightarrow M$ induces a morphism of exact sequences

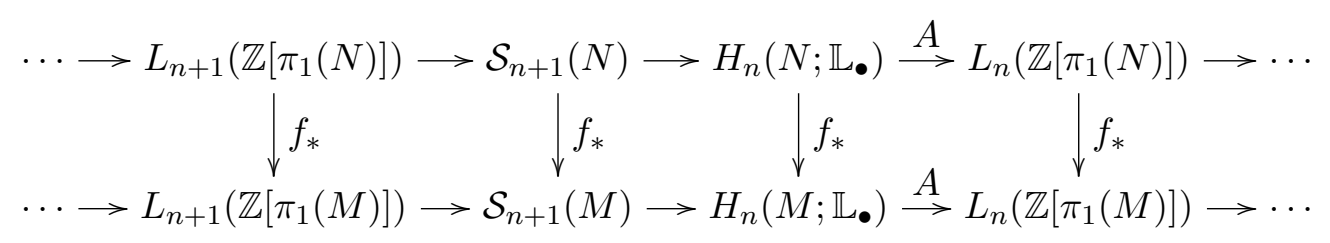

which is an isomorphism for a homotopy equivalence $f$. The algebraic and topological surgery sequences are isomorphic, with bijections

$$
\begin{aligned}
s: & \mathcal{S}^{T O P}(M) \rightarrow \mathcal{S}_{n+1}(M) ;(N, f) \mapsto s(f), \\
t & : \mathcal{T}^{T O P}(M)=[M, G / T O P] \rightarrow H_{n}\left(M ; \mathbb{L}_{\bullet}\right)
\end{aligned}
$$

which endow $\mathcal{S}^{T O P}(M)$ and $\mathcal{T}^{T O P}(M)$ with natural abelian group structures

$$
\begin{aligned}
& +: \mathcal{S}^{T O P}(M) \times \mathcal{S}^{T O P}(M) \rightarrow \mathcal{S}^{T O P}(M), \\
& +: \mathcal{T}^{T O P}(M) \times \mathcal{T}^{T O P}(M) \rightarrow \mathcal{T}^{T O P}(M) .
\end{aligned}
$$

The addition in $\mathcal{T}^{T O P}(M)$ corresponds to the disjoint union of normal maps. 
$\S 1$ recalls the algebraic and topological surgery exact sequences.

$\S 2$ uses the algebraic surgery exact sequence to prove the composition formula

$$
(P, f g)=(N, f)+f_{*}(P, g) \in \mathcal{S}^{T O P}(M)
$$

for homotopy equivalences $f: N \rightarrow M, g: P \rightarrow N$ of $n$-dimensional topological manifolds (Theorem 2.3). The addition in the structure set is thus given by

$$
(N, f)+\left(N^{\prime}, f^{\prime}\right)=(P, f g) \in \mathcal{S}^{T O P}(M)
$$

with $(P, g)=\left(f_{*}\right)^{-1}\left(N^{\prime}, f^{\prime}\right) \in \mathcal{S}^{T O P}(N)$.

A homotopy equivalence of $n$-dimensional manifolds $f: N \rightarrow M$ is automatically a normal map $(f, b)$, with

$$
b: \nu_{N} \rightarrow\left(f^{-1}\right)^{*} \nu_{N}=\nu_{M} \oplus \nu_{f} .
$$

Use the normal invariant $(f, b) \in[M, G / T O P]$ to define a function

$$
\eta: \mathcal{S}^{T O P}(M)=\mathcal{S}_{n+1}(M) \rightarrow \mathcal{T}^{T O P}(M) ;(N, f)=s(f) \mapsto \eta(N, f)=(f, b) .
$$

The composite of homotopy equivalences $f: N \rightarrow M, g: P \rightarrow N$ is a homotopy equivalence $f g: P \rightarrow M$ with normal invariant

$$
\eta(P, f g)=\eta(N, f) \oplus\left(f^{-1}\right)^{*} \eta(P, g) \in \mathcal{T}^{T O P}(M)
$$

by the sum formula of Brumfiel [1] and Madsen-Taylor-Williams [10].

At first sight, there is a disparity between the sum formulae $(*)$ and $(* *)$, since it is well-known that the disjoint union addition + and the Whitney sum addition $\oplus$ on the surgery classifying space $G / T O P \simeq \mathbb{L}_{0}$ are different, and the normal bordism set $\mathcal{T}^{T O P}(M)=[M, G / T O P]$ has two abelian group structures. A map $f: N \rightarrow M$ of $n$-dimensional manifolds induces a function $f_{*}: \mathcal{T}^{T O P}(N) \rightarrow \mathcal{T}^{T O P}(M)$ which is a morphism of abelian groups with respect to + , and a function $f^{*}: \mathcal{T}^{T O P}(M) \rightarrow \mathcal{T}^{T O P}(N)$ which is a morphism of abelian groups with respect to $\oplus$. If $f$ is a homotopy equivalence then both $f_{*}$ and $f^{*}$ are isomorphisms, with $\left(f_{*}\right)^{-1}=\left(f^{-1}\right)_{*},\left(f^{*}\right)^{-1}=\left(f^{-1}\right)^{*}$, but in general $f_{*}$ and $f^{*}$ are not inverse bijections. The diagram

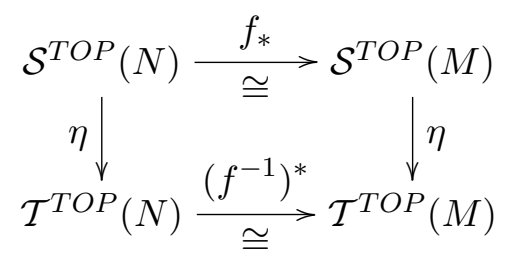


is not commutative, essentially since $f$ does not preserve the Hirzebruch $\mathcal{L}$-genus $\mathcal{L}(M) \in H_{n-4 *}(M ; \mathbb{Q})$

$$
f_{*} \mathcal{L}(N) \neq \mathcal{L}(M) \in H_{n-4 *}(M ; \mathbb{Q}) .
$$

An $n$-dimensional manifold $M$ has a canonical $\mathbb{L} \bullet$-theory fundamental class $[M]_{\mathbb{L}} \in$ $H_{n}\left(M ; \mathbb{L}^{\bullet}\right)$ with $\mathbb{L}^{\bullet}$ the symmetric $L$-theory spectrum of $\mathbb{Z}$ (Ranicki $[17, \S 16]$ ), such that

$$
[M]_{\mathbb{L}} \otimes \mathbb{Q}=\mathcal{L}(M) \in H_{n}\left(M ; \mathbb{L}^{\bullet}\right) \otimes \mathbb{Q}=H_{n-4 *}(M ; \mathbb{Q}) .
$$

The failure of commutativity in the diagram is traced in $\S 3$ to the failure (in general) of $f$ to preserve the symmetric $L$-theory fundamental classes, with

$$
f_{*}[N]_{\mathbb{L}}-[M]_{\mathbb{L}}=(1+T) \eta(N, f) \in \operatorname{im}\left(1+T: H_{n}\left(M ; \mathbb{L}_{\bullet}\right) \rightarrow H_{n}\left(M ; \mathbb{L}^{\bullet}\right)\right) .
$$

The normal invariant function $\eta: \mathcal{S}^{T O P}(M) \rightarrow \mathcal{T}^{T O P}(M)$ is a morphism of abelian groups with respect to + , so $(*)$ gives

$$
\eta(P, f g)=\eta(N, f)+\eta\left(f_{*}(P, g)\right) \in \mathcal{T}^{T O P}(M) .
$$

The symmetric $L$-theory orientation of manifolds is used in Corollary 3.5 to prove that

$$
\eta(N, f)+\eta\left(f_{*}(P, g)\right)=\eta(N, f) \oplus\left(f^{-1}\right)^{*} \eta(P, g) \in \mathcal{T}^{T O P}(M)
$$

reconciling $(*)$ and $(* *)$.

This paper is a substantial expansion of the paper posted on the arXiv in November 2006 (math.AT/0608705). I am grateful to Larry Taylor for asking me about the relationship between the manifold structure composition formula $\left(^{*}\right)$ and the normal invariant composition formula $\left({ }^{* *}\right)$ which stimulated me to expand the original version of the paper.

\section{The algebraic and topological surgery exaCt Sequences}

We refer to Wall [21] for the basic properties of geometric Poincaré complexes and pairs. A Spivak normal structure $\left(\nu_{M}, \rho_{M}\right)$ on an $n$-dimensional geometric Poincaré complex $M$ is a $(k-1)$-spherical fibration $\nu_{M}: M \rightarrow B G(k)$ together with a map $\rho_{M}: S^{n+k} \rightarrow T\left(\nu_{M}\right)$ to the Thom space with Hurewicz-Thom image the fundamental class

$$
h\left(\rho_{M}\right)=[M] \in \dot{H}_{n+k}\left(T\left(\nu_{M}\right)\right)=H_{n}(M) .
$$


Again, we refer to [21] for the existence and uniqueness properties of Spivak normal structures. An $n$-dimensional manifold $M$ is an $n$-dimensional geometric Poincaré complex. An embedding $M \subset S^{n+k}$ ( $k$ large) has a topological normal bundle $\nu_{M}: M \rightarrow B T O P(k)$ and the Pontrjagin-Thom construction gives a map $\rho_{M}: S^{n+k} \rightarrow T\left(\nu_{M}\right)$ such that $\left(J \nu_{M}, \rho_{M}\right)$ is a Spivak normal structure, with $J: B T O P(k) \rightarrow B G(k)$ the forgetful map. Similarly for geometric Poincaré pairs and manifolds with boundary.

A normal map $(f, b): N \rightarrow M$ of $n$-dimensional geometric Poincaré complexes with Spivak normal structures $\left(\nu_{N}, \rho_{N}\right),\left(\nu_{M}, \rho_{M}\right)$ is a degree 1 map $f: N \rightarrow M$ together with a map $b: \nu_{N} \rightarrow \nu_{M}$ of Spivak normal fibrations such that

$$
T(b) \rho_{N} \simeq \rho_{M}: S^{n+k} \rightarrow T\left(\nu_{M}\right) .
$$

A topological normal map $(f, b): N \rightarrow M$ of $n$-dimensional manifolds is a degree 1 map $f: N \rightarrow M$ together with a bundle map $b: \nu_{N} \rightarrow \nu_{M} \oplus \nu_{b}$ for some topological bundle $\nu_{b}: M \rightarrow B T O P(k)$ with a fibre homotopy trivialization $J \nu_{b} \simeq *: M \rightarrow B G(k)$, in which case $(f, J b): N \rightarrow M$ is a normal map of the underlying geometric Poincaré complexes with $J b: J \nu_{N} \rightarrow J \nu_{M}$. In particular, a homotopy equivalence $f: N \rightarrow M$ of manifolds determines a topological normal map $(f, b)$ with $b: \nu_{N} \rightarrow\left(f^{-1}\right)^{*} \nu_{N}$.

As usual, let

$$
G / T O P=\text { homotopy fibre of } J: B T O P \rightarrow B G,
$$

the classifying space for fibre homotopy trivialized stable topological bundles. A topological normal map $(f, b): N \rightarrow M$ has a topological normal invariant $\eta(f, b) \in[M, G / T O P]$ such that $b: \nu_{M} \rightarrow \nu_{N} \oplus \nu_{b}$ with

$$
\nu_{b}: M \stackrel{\eta(f, b)}{\longrightarrow} G / T O P \rightarrow B T O P .
$$

The normal map set $\mathcal{T}^{T O P}(M)$ of a manifold $M$ is the pointed set of bordism classes of topological normal maps $(f, b): N \rightarrow M$. The function

$$
\mathcal{T}^{T O P}(M) \rightarrow[M, G / T O P] ;(f, b) \mapsto \eta(f, b)
$$

is a bijection. The inverse is given by the Browder-Novikov transversality construction: given a map $\eta: M \rightarrow G / T O P$ let $\nu_{b}: M \rightarrow B T O P$ be the corresponding fibre homotopy trivial topological bundle, let $h_{\eta}: T\left(\nu_{M}\right) \simeq T\left(\nu_{M} \oplus \nu_{b}\right)$ 
be the corresponding homotopy equivalence of Thom spaces, and make the map

$$
h_{\eta} \rho_{M}: S^{n+k} \rightarrow T\left(\nu_{M}\right) \simeq T\left(\nu_{M} \oplus \nu_{b}\right)
$$

topologically transverse regular at the zero section $M \subset T\left(\nu_{M} \oplus \nu_{b}\right)$, to obtain a topological normal map

$$
(f, b)=h_{\eta} \rho_{M} \mid:\left(N, \nu_{N}\right)=\left(\left(h_{\eta} \rho_{M}\right)^{-1}(M), \nu_{N \subset S^{n+k}}\right) \rightarrow\left(M, \nu_{M} \oplus \nu_{b}\right)
$$

with topological normal invariant $\eta$. For a manifold with boundary $(M, \partial M)$ there is a relative normal map set $\mathcal{T}(M, \partial M)$ of topological normal maps $(f, b)$ : $(N, \partial N) \rightarrow(M, \partial M)$ with a bijection

$$
\mathcal{T}^{T O P}(M, \partial M) \cong[M, G / T O P] .
$$

There is also a rel $\partial$ normal map set $\mathcal{T}_{\partial}^{T O P}(M, \partial M)$ of topological normal maps $(f, b): N \rightarrow M$ such that $\partial f: \partial N \rightarrow \partial M$ is a homeomorphism, with a bijection

$$
\mathcal{T}_{\partial}^{T O P}(M, \partial M) \cong[M, \partial M ; G / T O P, *] .
$$

The Wall [22] surgery obstruction groups $L_{n}(\Lambda)$ of a ring $\Lambda$ with involution $\lambda \mapsto \bar{\lambda}$ were expressed in Ranicki [15] as the cobordism groups of $n$-dimensional quadratic Poincaré complexes $(C, \psi)$ over $\Lambda$, with $C$ an $n$-dimensional based f.g. free $\Lambda$-module chain complex. The quadratic structure is an element

$$
\psi \in Q_{n}(C)=H_{n}\left(W \otimes_{\mathbb{Z}\left[\mathbb{Z}_{2}\right]}\left(C \otimes_{\Lambda} C\right)\right)
$$

where

$$
W: \ldots \longrightarrow \mathbb{Z}\left[\mathbb{Z}_{2}\right] \stackrel{1-T}{\longrightarrow} \mathbb{Z}\left[\mathbb{Z}_{2}\right] \stackrel{1+T}{\longrightarrow} \mathbb{Z}\left[\mathbb{Z}_{2}\right] \stackrel{1-T}{\longrightarrow} \mathbb{Z}\left[\mathbb{Z}_{2}\right]
$$

is the standard free $\mathbb{Z}\left[\mathbb{Z}_{2}\right]$-module resolution of $\mathbb{Z}$, and $T \in \mathbb{Z}_{2}$ acts on

$$
C \otimes_{\Lambda} C=C \otimes_{\mathbb{Z}} C /\{x \otimes \lambda y-\bar{\lambda} x \otimes y \mid x, y \in C, \lambda \in \Lambda\}
$$

by

$$
T: C_{p} \otimes_{\Lambda} C_{q} \rightarrow C_{q} \otimes_{\Lambda} C_{p} ; x \otimes y \mapsto(-)^{p q} y \otimes x .
$$

The $\Lambda$-module chain map

$$
(1+T) \psi_{0}: C^{n-*}=\operatorname{Hom}_{\Lambda}(C, \Lambda)_{*-n} \rightarrow C
$$

is required to be a simple chain equivalence. The quadratic $L$-groups are the homotopy groups of an $\Omega$-spectrum $\mathbb{L}$ • $(\Lambda)$ of quadratic Poincaré complexes over $\Lambda$, with

$$
\pi_{n}\left(\mathbb{L}_{\bullet}(\Lambda)\right)=L_{n}(\Lambda)
$$


For the surgery exact sequence it is necessary to consider the 1-connective cover of $\mathbb{L} \bullet(\mathbb{Z})$, which is denoted by $\mathbb{L}_{\bullet}$, and is such that

$\mathbb{L}_{0} \simeq$ G/TOP,$\pi_{n}\left(\mathbb{L}_{\bullet}\right)=\pi_{n}(G / T O P)=L_{n}(\mathbb{Z})= \begin{cases}\mathbb{Z} & \text { if } n \equiv 0(\bmod 4) \\ 0 & \text { if } n \equiv 1(\bmod 4) \\ \mathbb{Z}_{2} & \text { if } n \equiv 2(\bmod 4) \\ 0 & \text { if } n \equiv 3(\bmod 4)\end{cases}$

The $H$-space structure on $\mathbb{L}_{0}$ given by the direct sum of quadratic Poincaré complexes corresponds to the disjoint union $H$-space structure on $G / T O P$.

The symmetric L-groups $L^{n}(\Lambda)$ of a ring $\Lambda$ with involution were defined in [15] (following Mishchenko) as the cobordism groups of $n$-dimensional symmetric Poincaré complexes $(C, \phi)$ over $\Lambda$, with $C$ an $n$-dimensional based f.g. free $\Lambda$ module chain complex. The symmetric structure is an element

$$
\phi \in Q^{n}(C)=H_{n}\left(\operatorname{Hom}_{\mathbb{Z}\left[\mathbb{Z}_{2}\right]}\left(W, C \otimes_{\Lambda} C\right)\right)
$$

and the $\Lambda$-module chain map $\phi_{0}: C^{n-*} \rightarrow C$ is required to be a simple chain equivalence. The symmetrization maps

$$
1+T: L_{n}(\Lambda) \rightarrow L^{n}(\Lambda) ;(C, \psi) \mapsto(C,(1+T) \psi)
$$

are isomorphisms modulo 8-torsion ([14, Proposition 8.2]). For any rings with involution $\Lambda, \Lambda^{\prime}$ the tensor product over $\mathbb{Z}$ defines $L$-theory products

$$
\begin{aligned}
& L^{n}(\Lambda) \otimes_{\mathbb{Z}} L^{n^{\prime}}\left(\Lambda^{\prime}\right) \rightarrow L^{n+n^{\prime}}\left(\Lambda \otimes_{\mathbb{Z}} \Lambda^{\prime}\right), \\
& L^{n}(\Lambda) \otimes_{\mathbb{Z}} L_{n^{\prime}}\left(\Lambda^{\prime}\right) \rightarrow L_{n+n^{\prime}}\left(\Lambda \otimes_{\mathbb{Z}} \Lambda^{\prime}\right)
\end{aligned}
$$

and also

$$
L_{n}(\Lambda) \otimes_{\mathbb{Z}} L_{n^{\prime}}\left(\Lambda^{\prime}\right) \rightarrow L_{n+n^{\prime}}\left(\Lambda \otimes_{\mathbb{Z}} \Lambda^{\prime}\right) ; x \otimes x^{\prime} \mapsto(1+T) x \otimes x^{\prime}=x \otimes(1+T) x^{\prime} .
$$

The symmetric signature of an $n$-dimensional geometric Poincaré complex $M$ was defined in Ranicki [16] (again, following Mishchenko) to be the cobordism class

$$
\sigma^{*}(M)=\left(C(\widetilde{M}), \phi_{M}\right) \in L^{n}\left(\mathbb{Z}\left[\pi_{1}(M)\right]\right)
$$

of the $n$-dimensional symmetric Poincaré complex $\left(C(\widetilde{M}), \phi_{M}\right)$ over $\mathbb{Z}\left[\pi_{1}(M)\right]$ with $\widetilde{M}$ is the universal cover of $M$. The symmetric $L$-groups are the homotopy groups of an $\Omega$-spectrum $\mathbb{L} \bullet(\Lambda)$ of symmetric Poincaré complexes over $\Lambda$, with

$$
\pi_{n}\left(\mathbb{L}^{\bullet}(\Lambda)\right)=L^{n}(\Lambda) .
$$


In particular, for $\Lambda=\mathbb{Z}$ we write $\mathbb{L} \bullet(\mathbb{Z})=\mathbb{L} \bullet$, with

$$
\pi_{n}\left(\mathbb{L}^{\bullet}\right)=L^{n}(\mathbb{Z})= \begin{cases}\mathbb{Z} & \text { if } n \equiv 0(\bmod 4) \\ \mathbb{Z}_{2} & \text { if } n \equiv 1(\bmod 4) \\ 0 & \text { if } n \equiv 2(\bmod 4) \\ 0 & \text { if } n \equiv 3(\bmod 4)\end{cases}
$$

The tensor product of symmetric Poincaré complexes over $\mathbb{Z}$ gives $\mathbb{L} \bullet$ the structure of a ring spectrum (with a 1 ), $\mathbb{L}_{\bullet}(\Lambda)$ is an $\mathbb{L}^{\bullet}$-module spectrum, and $\mathbb{L}_{\bullet}$ is also a ring spectrum (but only with an 8 , not a 1 ). A topological bundle has an $\mathbb{L}^{\bullet}$-orientation, and an $n$-dimensional manifold $M$ has a symmetric $L$-theory fundamental class $[M]_{\mathbb{L}} \in H_{n}\left(M ; \mathbb{L}^{\bullet}\right)([17$, Proposition 16.16]) with assembly

$$
A\left([M]_{\mathbb{L}}\right)=\sigma^{*}(M) \in L^{n}\left(\mathbb{Z}\left[\pi_{1}(M)\right]\right) .
$$

The quadratic signature of a normal map $(f, b): N \rightarrow M$ of $n$-dimensional geometric Poincaré complexes was defined in Ranicki [16] to be the cobordism class

$$
\sigma_{*}(f, b)=\left(\mathcal{C}\left(f^{!}\right), \psi_{b}\right) \in L_{n}\left(\mathbb{Z}\left[\pi_{1}(M)\right]\right)
$$

of the quadratic kernel $n$-dimensional quadratic Poincaré complex $\left(\mathcal{C}\left(f^{!}\right), \psi_{b}\right)$ over $\mathbb{Z}\left[\pi_{1}(M)\right]$, constructed as follows. The Umkehr $\mathbb{Z}\left[\pi_{1}(M)\right]$-module chain map is the composite

$$
f^{!}: C(\widetilde{M}) \simeq C(\widetilde{M})^{n-*} \stackrel{\widetilde{f}^{*}}{\longrightarrow} C(\widetilde{N})^{n-*} \simeq C(\widetilde{N})
$$

where $\widetilde{M}$ is the universal cover of $M, \widetilde{N}=f^{*} \widetilde{M}$ is the pullback cover of $N$, and $\widetilde{f}: \widetilde{N} \rightarrow \widetilde{M}$ is a $\pi_{1}(M)$-equivariant lift of $f$. The algebraic mapping cone $\mathcal{C}\left(f^{!}\right)$ fits into the chain homotopy direct sum system

$$
C(\widetilde{M}) \underset{\widetilde{f}}{\stackrel{f^{!}}{\rightleftarrows}} C(\widetilde{N}) \stackrel{e}{\rightleftarrows} \mathcal{C}\left(f^{!}\right)
$$

with $e: C(\widetilde{N}) \rightarrow \mathcal{C}\left(f^{!}\right)$the inclusion and $\widetilde{f} f^{!} \simeq 1: C(\widetilde{M}) \rightarrow C(\widetilde{M})$. Thus there is defined a chain equivalence of symmetric Poincaré complexes

$$
\left(C(\widetilde{N}), \phi_{N}\right) \simeq\left(\mathcal{C}\left(f^{!}\right),(1+T) \psi_{b}\right) \oplus\left(C(\widetilde{M}), \phi_{M}\right),
$$


and

$$
\begin{aligned}
& H_{*}\left(\mathcal{C}\left(f^{!}\right)\right)=K_{*}(N)=\operatorname{ker}\left(\widetilde{f}_{*}: H_{*}(\widetilde{N}) \rightarrow H_{*}(\widetilde{M})\right) \\
& H_{*}(\widetilde{N})=H_{*}\left(\mathcal{C}\left(f^{!}\right)\right) \oplus H_{*}(\widetilde{M}) .
\end{aligned}
$$

Let $\nu_{\widetilde{M}}$ be the pullback of $\nu_{M}$ to the universal cover $\widetilde{M}$ of $M$, and let $\nu_{\widetilde{N}}$ be the pullback of $\nu_{N}$ to the pullback cover $\widetilde{N}=f^{*} \widetilde{M}$ of $N$, so that $b$ lifts to a $\pi_{1}(M)$ equivariant map $\widetilde{b}: \nu_{\widetilde{N}} \rightarrow \nu_{\widetilde{M}}$. The $\pi_{1}(M)$-equivariant $S$-dual of $T(\widetilde{b}): T\left(\nu_{\widetilde{N}}\right) \rightarrow$ $T\left(\nu_{\widetilde{M}}\right)$ is a $\pi_{1}(M)$-equivariant stable map $F: \Sigma^{\infty} \widetilde{M}^{+} \rightarrow \Sigma^{\infty} \widetilde{N}^{+}$inducing $f^{!}$on the chain level. The quadratic construction of $[16, \S 1]$ is a natural transformation

$$
\psi_{F}: C(M) \rightarrow W \otimes_{\mathbb{Z}\left[\mathbb{Z}_{2}\right]}\left(C(\tilde{N}) \otimes_{\mathbb{Z}\left[\pi_{1}(M)\right]} C(\tilde{N})\right)
$$

and the quadratic structure in $\sigma_{*}(f, b)$ is defined by

$$
\psi_{b}=(e \otimes e) \psi_{F}[M] \in Q_{n}\left(\mathcal{C}\left(f^{!}\right)\right) .
$$

There is also a version for a normal map of geometric Poincaré pairs.

The surgery obstruction $\sigma_{*}(f, b) \in L_{n}\left(\mathbb{Z}\left[\pi_{1}(M)\right]\right)$ was defined by Wall [22] for a topological $n$-dimensional normal map $(f, b): N \rightarrow M$ of manifolds with boundary such that $\partial f: \partial N \rightarrow \partial M$ is a homotopy equivalence. The surgery obstruction was identified in [16] with the quadratic signature of the underlying normal map of geometric Poincaré pairs.

The algebraic normal invariant of a topological normal map $(f, b): N \rightarrow M$ of $n$-dimensional manifolds is the cobordism class

$$
t(f, b)=(C, \psi) \in H_{n}\left(M ; \mathbb{L}_{\bullet}\right)
$$

defined in [17, Proposition 18.3] (as recalled in $\S 2$ below), with symmetrization

$$
(1+T) t(f, b)=f_{*}[N]_{\mathbb{L}}-[M]_{\mathbb{L}} \in H_{n}\left(M ; \mathbb{L}_{\bullet}\right)
$$

and assembly the surgery obstruction

$$
A(t(f, b))=\sigma_{*}(f, b) \in L_{n}\left(\mathbb{Z}\left[\pi_{1}(M)\right]\right) .
$$

The generalized homology group $H_{n}\left(X ; \mathbb{L}_{\bullet}\right)$ of any space $X$ can be identified with the bordism group of $n$-dimensional topological normal maps $(f, b): N \rightarrow M$ with a reference map $g: M \rightarrow X:$ the bordism class can be identified with $g_{*} t(f, b) \in H_{n}\left(X ; \mathbb{L}_{\bullet}\right)$. From this point of view the addition is by disjoint union

$$
\begin{aligned}
& ((f, b): N \rightarrow M, g: M \rightarrow X)+\left(\left(f^{\prime}, b^{\prime}\right): N^{\prime} \rightarrow M^{\prime}, g^{\prime}: M^{\prime} \rightarrow X\right) \\
& \quad=\left((f, b) \sqcup\left(f^{\prime}, b^{\prime}\right): N \sqcup N^{\prime} \rightarrow M \sqcup M^{\prime}, g \sqcup g^{\prime}: M \sqcup M^{\prime} \rightarrow X\right) \in H_{n}\left(X: \mathbb{L}_{\bullet}\right) .
\end{aligned}
$$


The structure set $\mathcal{S}^{T O P}(M)$ of an $n$-dimensional topological manifold $M$ was already defined in the Introduction. For a manifold with boundary $(M, \partial M)$ there is a relative structure set $\mathcal{S}^{T O P}(M, \partial M)$ of equivalence classes of pairs $(N, f)$ with $N$ an $n$-dimensional manifold with boundary and $f: N \rightarrow M$ a homotopy equivalence. There is also a rel $\partial$ structure set $\mathcal{S}_{\partial}^{T O P}(M, \partial M)$ of equivalence classes of pairs $(N, f)$ with $N$ an $n$-dimensional manifold with boundary and $f$ : $N \rightarrow M$ a homotopy equivalence such that $\partial f: \partial N \rightarrow \partial M$ is a homeomorphism.

For $n \geqslant 5$ and an $n$-dimensional manifold $M$ the Browder-Novikov-SullivanWall surgery theory (extended to the topological category by Kirby and Siebenmann [7]) fits $\mathcal{S}_{\partial}^{T O P}\left(M \times D^{k}\right)(k \geqslant 0)$ into an exact sequence of pointed sets

$$
\begin{aligned}
\ldots \longrightarrow & \mathcal{S}_{\partial}^{T O P}\left(M \times D^{k}\right) \stackrel{\eta}{\longrightarrow} \mathcal{T}_{\partial}^{T O P}\left(M \times D^{k}\right) \stackrel{\theta}{\longrightarrow} L_{n+k}\left(\mathbb{Z}\left[\pi_{1}(M)\right]\right) \longrightarrow \\
\ldots & \longrightarrow \mathcal{S}_{\partial}^{T O P}\left(M \times D^{1}\right) \stackrel{\eta}{\longrightarrow} \mathcal{T}_{\partial}^{T O P}\left(M \times D^{1}\right) \stackrel{\theta}{\longrightarrow} L_{n+1}\left(\mathbb{Z}\left[\pi_{1}(M)\right]\right) \\
& \longrightarrow \mathcal{S}^{T O P}(M) \stackrel{\eta}{\longrightarrow} \mathcal{T}^{T O P}(M) \stackrel{\theta}{\longrightarrow} L_{n}\left(\mathbb{Z}\left[\pi_{1}(M)\right]\right)
\end{aligned}
$$

which is in fact an exact sequence of abelian groups until $L_{n+1}\left(\mathbb{Z}\left[\pi_{1}(M)\right]\right)$, with $\mathcal{T}_{\partial}^{T O P}\left(M \times D^{k}\right)=\left[M \times D^{k}, \partial ; G / T O P, *\right]$, using the disjoint union $H$-space structure on $G / T O P$. For a manifold with boundary $(M, \partial M)$ there are also rel and rel $\partial$ versions. See Wall [22, Theorem 10.8], Quinn [12, 13], Siebenmann [7, Essay V, Appendix C], Ranicki [15, 16], Nicas [11], Cappell and Weinberger [2] and Kro [9] for previous accounts of the topological structure set.

The quadratic $\mathcal{S}$-groups $\mathcal{S}_{*}(X)$ of a space $X$ were defined in $[17, \S 15]$, to fit into the algebraic surgery exact sequence

$$
\cdots \rightarrow H_{n}\left(X ; \mathbb{L}_{\bullet}\right) \stackrel{A}{\rightarrow} L_{n}\left(\mathbb{Z}\left[\pi_{1}(X)\right]\right) \rightarrow \mathcal{S}_{n}(X) \rightarrow H_{n-1}\left(X ; \mathbb{L}_{\bullet}\right) \rightarrow \cdots
$$

with $A$ the assembly map. The functor

$$
\{\text { spaces }\} \rightarrow\{\mathbb{Z} \text {-graded abelian groups }\} ; X \mapsto \mathcal{S}_{*}(X)
$$

is covariant and homotopy invariant: a map $f: X \rightarrow Y$ induces morphisms $f_{*}: \mathcal{S}_{*}(X) \rightarrow \mathcal{S}_{*}(Y)$ depending only on the homotopy class of $f$. A homotopy equivalence $f$ induces isomorphisms $f_{*}$.

Proposition 1.1. (Ranicki [17, Theorem 18.5]) The algebraic and topological surgery exact sequences of an $n$-dimensional manifold $M$ with $n \geqslant 5$ are related 
by an isomorphism

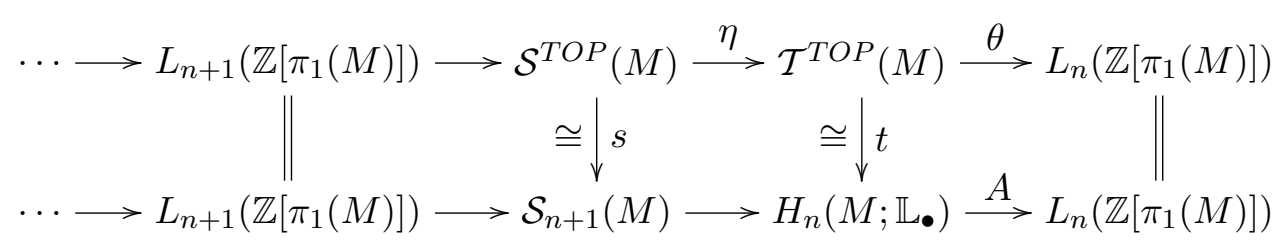

Similarly for a manifold with boundary.

Proof. The isomorphism sending the topological normal invariant to the algebraic normal invariant

$$
\begin{gathered}
t=[M]_{\mathbb{L}} \cap-: \mathcal{T}^{T O P}(M)=[M, G / T O P]=H^{0}\left(M ; \mathbb{L}_{\bullet}\right) \stackrel{\cong}{\longrightarrow} H_{n}\left(M ; \mathbb{L}_{\bullet}\right) ; \\
\eta(f, b) \mapsto t(f, b)=[M]_{\mathbb{L}} \cap \eta(f, b)
\end{gathered}
$$

is the Poincaré duality isomorphism defined by cap product with the symmetric $L$-theory fundamental class $[M]_{\mathbb{L}} \in H_{n}\left(M ; \mathbb{L}^{\bullet}\right)$, sending the topological normal invariant of a normal map $(f, b): N \rightarrow M$ to the algebraic normal invariant

$$
t(f, b)=[M]_{\mathbb{L}} \cap \eta(f, b) \in H_{n}\left(M ; \mathbb{L}_{\bullet}\right) .
$$

Here, we are using the disjoint union $H$-space structure on $G / T O P$ to define the abelian group structure, which corresponds to the direct sum of quadratic Poincaré complexes in $\mathbb{L}_{\bullet}$. The composite of $t$ and the quadratic $L$-theory assembly map $A$

$$
\text { At }: \mathcal{T}^{T O P}(M) \cong H_{n}\left(M ; \mathbb{L}_{\bullet}\right) \rightarrow L_{n}\left(\mathbb{Z}\left[\pi_{1}(M)\right]\right)
$$

sends a topological normal map $(f, b): N \rightarrow M$ to the surgery obstruction

$$
A t(f, b)=\sigma_{*}(f, b) \in L_{n}\left(\mathbb{Z}\left[\pi_{1}(M)\right]\right) .
$$

The isomorphism

$$
s: \mathcal{S}^{T O P}(M) \stackrel{\cong}{\longrightarrow} \mathcal{S}_{n+1}(M) ;(N, f) \mapsto s(f)
$$

sends a homotopy equivalence $f: N \rightarrow M$ to the 'manifold structure' $s(f)$ measuring the failure of the point inverses $f^{-1}(x) \subset N(x \in M)$ to be acyclic. (At this point it is worth recalling the $C E$ approximation theorem of Siebenmann [20]: for $n \geqslant 5$ a map of $n$-dimensional topological manifolds $f: N \rightarrow M$ with contractible point inverses is a homotopy equivalence which is homotopic to a homeomorphism). 
Remark 1.2. Proposition 1.1 also applies in dimension $n=4$ in the case of a fundamental group $\pi_{1}(M)$ which is good in the sense of Freedman and Quinn $[4]$.

\section{Composition formulae}

The composition formula for manifold structures is just a local version of the composition formula of Ranicki [16] for surgery obstructions, so we start with that:

Proposition 2.1. ([16, Proposition 4.3]) The composite of normal maps of $n$ dimensional geometric Poincaré complexes

$$
(g, c): P \rightarrow N,(f, b): N \rightarrow M
$$

is a normal map $(f g, b c): P \rightarrow M$ with surgery obstruction

$$
\sigma_{*}(f g, b c)=\sigma_{*}(f, b)+f_{*} \sigma_{*}(g, c) \in L_{n}\left(\mathbb{Z}\left[\pi_{1}(M)\right]\right) .
$$

Proof. Homotopy equivalent quadratic Poincaré complexes are cobordant. The Umkehr chain maps are such that up to chain homotopy

$$
(f g)^{!}=g^{!} f^{!}: C(\widetilde{M}) \stackrel{f^{!}}{\longrightarrow} C(\widetilde{N}) \stackrel{g^{!}}{\longrightarrow} C(\widetilde{P})
$$

and the quadratic kernels are such that up homotopy equivalence

$$
\left(\mathcal{C}\left((f g)^{!}\right), \psi_{b c}\right)=\left(\mathcal{C}\left(f^{!}\right), \psi_{b}\right) \oplus f_{*}\left(\mathcal{C}\left(g^{!}\right), \psi_{c}\right) .
$$

Remark 2.2. The surgery composition formula of Proposition 2.1 avoids the framing problems of the surgery composition formulae for topological normal maps of Wall [22, p.279] and Jones [6, 7.0], being purely homotopy theoretic in nature. See Proposition 2.7 below for the composition of topological normal maps.

The generalized $\mathbb{L}_{\bullet}$-homology groups $H_{*}\left(X ; \mathbb{L}_{\bullet}\right)$ of a finite simplicial complex $X$ were identified in Ranicki $[17, \S 12]$ with the quadratic $L$-groups of an additive category $\mathbb{A}(\mathbb{Z}, X)$ with chain duality in which a quadratic Poincaré complex is a sheaf of quadratic Poincaré complexes over $X$, i.e. satisfies local Poincaré duality. 
As in Ranicki and Weiss [19] define a $(\mathbb{Z}, X)$-module $A$ to be a $\mathbb{Z}$-module with a direct sum decomposition

$$
A=\sum_{\sigma \in X} A(\sigma)
$$

with each $A(\sigma)$ based f.g. free. Let $\mathbb{A}(\mathbb{Z}, X)$ be the additive category with objects $(\mathbb{Z}, X)$-modules and morphisms $f: A \rightarrow B$ the abelian group morphisms such that

$$
f(A(\sigma)) \subseteq \sum_{\tau \geqslant \sigma} B(\tau) .
$$

As in $[17, \S \S 5,13],[18]$ there is defined a chain duality on $\mathbb{A}(\mathbb{Z}, X)$ such that

$$
H_{n}\left(X ; \mathbb{L}_{\bullet}\right)=L_{n}(\mathbb{A}(\mathbb{Z}, X)) .
$$

An element $(C, \psi) \in H_{n}\left(X ; \mathbb{L}_{\bullet}\right)$ is a cobordism class of $n$-dimensional quadratic Poincaré complexes $(C, \psi)$ in $\mathbb{A}(\mathbb{Z}, X)$ with $C(\sigma)$ an $(n-|\sigma|)$-dimensional based f.g. free $\mathbb{Z}$-module chain complex, subject to the additional requirement (corresponding to the 1 -connectivity of $\mathbb{L}_{\bullet}$ ) that $C(\sigma)$ be contractible for $\sigma \in C$ with $|\sigma|=n$. The quadratic structure is an element

$$
\psi \in Q_{n}^{X}(C)=H_{n}\left(W \otimes_{\mathbb{Z}\left[\mathbb{Z}_{2}\right]}\left(C \otimes_{\mathbb{A}(\mathbb{Z}, X)} C\right)\right)
$$

with $C \otimes_{\mathbb{A}(\mathbb{Z}, X)} C \subseteq C \otimes_{\mathbb{Z}} C$ the $\mathbb{Z}\left[\mathbb{Z}_{2}\right]$-module subcomplex defined by

$$
C \otimes_{\mathbb{A}(\mathbb{Z}, X)} C=\sum_{\sigma, \tau \in X, \sigma \cap \tau \neq \emptyset} C(\sigma) \otimes_{\mathbb{Z}} C(\tau) .
$$

Let $\mathbb{A}\left(\mathbb{Z}\left[\pi_{1}(X)\right]\right)$ be the additive category of based f.g. free $\mathbb{Z}\left[\pi_{1}(X)\right]$-modules. The assembly functor $([19],[17, \S 9])$

$$
A: \mathbb{A}(\mathbb{Z}, X) \rightarrow \mathbb{A}\left(\mathbb{Z}\left[\pi_{1}(X)\right]\right) ; B=\sum_{\sigma \in X} B(\sigma) \mapsto A(B)=\sum_{\widetilde{\sigma} \in \widetilde{X}} B(p \widetilde{\sigma})
$$

was defined using the universal covering projection $p: \widetilde{X} \rightarrow X$. An element $(C, \psi) \in H_{n}\left(X ; \mathbb{L}_{\bullet}\right)$ is a cobordism class of $n$-dimensional quadratic Poincaré complexes $(C, \psi)$ in $\mathbb{A}(\mathbb{Z})_{*}(X)$. An element $(C, \psi) \in \mathcal{S}_{n+1}(X)$ is a cobordism class of $n$-dimensional quadratic Poincaré complexes $(C, \psi)$ in $\mathbb{A}(\mathbb{Z})_{*}(X)$ such that the assembly based f.g. free $\mathbb{Z}\left[\pi_{1}(X)\right]$-module chain complex $A(C)$ is simple contractible.

We now recall from [17] the construction of the algebraic normal invariant $t(f, b) \in H_{n}\left(M ; \mathbb{L}_{\bullet}\right)$ of a topological normal map $(f, b): N \rightarrow M$ of $n$-dimensional manifolds. By [17, Corollary 17.6] there exists a finite simplicial complex $X$ with 
a homotopy equivalence $h: M \rightarrow X$ such that both $h$ and $h f: N \rightarrow X$ are topologically transverse across the dual cell decomposition $\{D(\sigma, X) \mid \sigma \in X\}$ of the barycentric subdivision $X^{\prime}$. The $\mathbb{L}_{\bullet}$-homology groups are homotopy invariant, so $h_{*}: H_{n}\left(M ; \mathbb{L}_{\bullet}\right) \rightarrow H_{n}\left(X ; \mathbb{L}_{\bullet}\right)$ is an isomorphism. The restrictions

$$
(f(\sigma), b(\sigma))=(f, b) \mid:(h f)^{-1} D(\sigma, X) \rightarrow h^{-1} D(\sigma, X)(\sigma \in X)
$$

are normal maps of $(n-|\sigma|)$-dimensional geometric Poincaré pairs (in fact manifolds) with boundaries the inverse images of the boundaries of the dual cells

$$
\partial D(\sigma, X)=\bigcup_{\tau>\sigma} D(\tau, X) \subset D(\sigma, X),
$$

such that the degree 1 map is the assembly

$$
f=\bigcup_{\sigma \in X} f(\sigma): N=\bigcup_{\sigma \in X}(h f)^{-1} D(\sigma, X) \rightarrow M=\bigcup_{\sigma \in X} h^{-1} D(\sigma, X) .
$$

The quadratic cycle $(C, \psi)$ has chain components

$$
C(\sigma)=\mathcal{C}\left(f(\sigma)^{!}: C\left(h^{-1} D(\sigma, X)\right) \rightarrow C\left((h f)^{-1} D(\sigma, X)\right)\right)(\sigma \in X)
$$

with assembly the based f.g. free $\mathbb{Z}\left[\pi_{1}(X)\right]$-module chain complex

$$
A(C)=\mathcal{C}\left(\widetilde{f}^{!}: C(\widetilde{M}) \rightarrow C(\widetilde{N})\right),
$$

and the quadratic components $\psi(\sigma)$ are the quadratic structures of the normal maps $(f(\sigma), b(\sigma))$ used to define the algebraic normal invariant

$$
t(f, b)=(C, \psi) \in H_{n}\left(X ; \mathbb{L}_{\bullet}\right)=H_{n}\left(M ; \mathbb{L}_{\bullet}\right) .
$$

The algebraic manifold structure of a homotopy equivalence $f: N \rightarrow M$ of $n$-dimensional manifolds is the cobordism class

$$
s(f)=(C, \psi) \in \mathcal{S}_{n+1}(X)=\mathcal{S}_{n+1}(M)
$$

with $(C, \psi)$ (constructed as above) simple $\mathbb{Z}\left[\pi_{1}(M)\right]$-contractible by virtue of $f$ being a homotopy equivalence. The bijection of Proposition 1.1

$$
\mathcal{S}^{T O P}(M) \rightarrow \mathcal{S}_{n+1}(M) ;(N, f) \mapsto s(f)=(C, \psi)
$$

sends the topological manifold structure to the algebraic manifold structure.

Theorem 2.3. Let $n \geqslant 5$. The manifold structure of the composite $f g: P \rightarrow M$ of homotopy equivalences $g: P \rightarrow N, f: N \rightarrow M$ of $n$-dimensional manifolds is given by

$$
s(f g)=s(f)+f_{*} s(g) \in \mathcal{S}^{T O P}(M)=\mathcal{S}_{n+1}(M) .
$$


Proof. Let $X$ be a finite simplicial complex with a homotopy equivalence $h$ : $M \rightarrow X$ such that $h$ and $h f: N \rightarrow X$ and $h f g: P \rightarrow X$ are topologically transverse across the dual cell decomposition $\{D(\sigma, X) \mid \sigma \in X\}$ of the barycentric subdivision $X^{\prime}$. Exactly as in the proof of Proposition 2.1 the chain complex kernels of the composite normal maps

$$
\begin{aligned}
& ((f g)(\sigma),(b c)(\sigma))=(f(\sigma), b(\sigma))(g(\sigma), c(\sigma)): \\
& \quad(h f g)^{-1} D(\sigma, X) \rightarrow(h f)^{-1} D(\sigma, X) \rightarrow h^{-1} D(\sigma, X)(\sigma \in X)
\end{aligned}
$$

split (up to $\mathbb{Z}$-module chain equivalence) as direct sums

$$
\mathcal{C}\left((f g)(\sigma)^{!}\right)=\mathcal{C}\left(f(\sigma)^{!}\right) \oplus \mathcal{C}\left(g(\sigma)^{!}\right)
$$

and similarly for the quadratic structures $\psi$. It follows that

$$
s(f g)=s(f)+f_{*} s(g) \in \mathcal{S}^{T O P}(M)=\mathcal{S}_{n+1}(M)=\mathcal{S}_{n+1}(X)
$$

completing the proof.

Remark 2.4. (i) Theorem 2.3 also applies in dimension $n=4$ in the case of a fundamental group $\pi_{1}(M)$ which is good in the sense of Freedman and Quinn [4]. (ii) Theorem 2.3 is in fact true for all $n \geqslant 0$, provided that $f_{*}(P, g) \in \mathcal{S}^{T O P}(M)$ is interpreted as an element of $\mathcal{S}_{n+1}(M)$ rather than as a manifold structure. The algebraic manifold structure $s(f) \in \mathcal{S}_{n+1}(M)$ is defined for any homotopy equivalence $f: N \rightarrow M$ of $n$-dimensional manifolds, and the proof of 2.3 gives $s(f g)=s(f)+f_{*} s(g) \in \mathcal{S}_{n+1}(M)$.

For any degree 1 map $f: N \rightarrow M$ of $n$-dimensional geometric Poincaré complexes there is defined a commutative square

$$
\begin{gathered}
H^{r}(N)<\frac{f^{*}}{} H^{r}(M) \\
{[N] \cap-\downarrow \cong f_{*}[N] \cap-} \\
H_{n-r}(N) \stackrel{f_{*}}{\longrightarrow} H_{n-r}(M)
\end{gathered}
$$

with $f_{*}[N]=[M] \in H_{n}(M)$, so that the induced morphisms $f^{*}: H^{r}(N) \rightarrow$ $H^{r}(M)$ are split injections. Similarly: 
Lemma 2.5. For a topological normal map $(f, b): N \rightarrow M$ of $n$-dimensional manifolds there is defined a commutative square

$$
\begin{aligned}
& {[N, G / T O P]=H^{0}\left(N ; \mathbb{L}_{\bullet}\right) \stackrel{f^{*}}{\longleftarrow}[M, G / T O P]=H^{0}\left(M ; \mathbb{L}_{\bullet}\right)} \\
& \begin{aligned}
{[N]_{\mathbb{L}} \cap-\downarrow } & & \cong \downarrow f_{*}[N]_{\mathbb{L}} \cap- \\
H_{n}\left(N ; \mathbb{L}_{\bullet}\right) & f_{*} & \longrightarrow H_{n}\left(M ; \mathbb{L}_{\bullet}\right)
\end{aligned}
\end{aligned}
$$

so that

$$
f^{*}:[M, G / T O P] \rightarrow[N, G / T O P] ; \eta \mapsto f^{*} \eta
$$

is a split injection.

Remark 2.6. (i) A degree 1 map of manifolds $f: N \rightarrow M$ does not in general have degree 1 in $\mathbb{L}^{\bullet}$-homology

$$
f_{*}[N]_{\mathbb{L}} \neq[M]_{\mathbb{L}} \in H_{n}\left(M ; \mathbb{L}^{\bullet}\right) .
$$

(ii) If $(f, b): N \rightarrow M$ is a topological normal map then

$$
f_{*}[N]_{\mathbb{L}}=[M]_{\mathbb{L}}+(1+T) t(f, b) \in H_{n}\left(M ; \mathbb{L}^{\bullet}\right)
$$

is an $\mathbb{L}^{\bullet}$-theory fundamental class.

In general, it is not possible to compose topological normal maps. However:

Proposition 2.7. For topological normal maps $(f, b): N \rightarrow M,(g, c): P \rightarrow N$ such that

$$
\eta(g, c) \in \operatorname{im}\left(f^{*}:[M, G / T O P] \rightarrow[N, G / T O P]\right)
$$

it is possible to define the composite topological normal map $(f g, b c): P \rightarrow M$ with

$$
b c: \nu_{P} \stackrel{c}{\longrightarrow} \nu_{N} \oplus \nu_{c} \stackrel{b \oplus 1}{\longrightarrow} \nu_{M} \oplus \nu_{b} \oplus f_{!} \nu_{c},
$$

with normal invariant $f_{!} \eta(g, c) \in[M, G / T O P]$ the unique element such that

$$
\eta(g, c)=f^{*}\left(f_{!} \eta(g, c)\right) \in[N, G / T O P] .
$$

(i) The topological normal invariant of the composite is given by

$$
\eta(f g, b c)=\eta(f, b) \oplus f_{!} \eta(g, c) \in[M, G / T O P] .
$$

(ii) The algebraic normal invariant of the composite is given by

$$
t(f g, b c)=t(f, b)+f_{*} t(g, c) \in H_{n}\left(M ; \mathbb{L}_{\bullet}\right) .
$$


Proof. (i) By construction.

(ii) Exactly as for the proof of Theorem 2.3.

Remark 2.8. In particular, the hypothesis of Proposition 2.7 is satisfied in the special case when $f: N \rightarrow M$ is a homotopy equivalence, with

$$
f_{!} \eta(g, c)=\left(f^{-1}\right)^{*} \eta(g, c) \in[M, G / T O P] .
$$

The composition formula in this case

$$
\eta(f g, b c)=\eta(f, b) \oplus\left(f^{-1}\right)^{*} \eta(g, c) \in[M, G / T O P]
$$

was first obtained by Brumfiel [1, Proposition 2.2] and Madsen, Taylor and Williams [10, Lemma 2.5].

In order to reconcile the composition formulae of Proposition 2.7 for the topological and algebraic normal invariants it is necessary deal with the bijection

$$
\eta: \mathcal{T}^{T O P}(M)=[M, G / T O P] \rightarrow H_{n}\left(M ; \mathbb{L}_{\bullet}\right) ; \eta(f, b) \mapsto t(f, b) .
$$

and compare the Whitney sum addition on the left with the direct sum addition on the right, as will now be done in $\S 3$.

\section{The Whitney sum And Products}

It has long been known that the surgery obstruction function

$$
\theta: \mathcal{T}^{T O P}(M)=[M, G / T O P] \rightarrow L_{n}\left(\mathbb{Z}\left[\pi_{1}(M)\right]\right)
$$

is a function between abelian groups which is not a morphism. This was first observed for $P L$ normal invariants in the simply-connected case by Cooke [3, p.182], and in general by Wall [22, p.114] :

We enter here a caveat to the reader. In the situation of (10.6), we have the map

$$
\theta:[X, G / P L] \rightarrow L_{m}(\pi(X))
$$

of abelian groups which satisfies, by definition, $\theta(0)=0$. However, $\theta$ is NOT in general a homomorphism. The result fails even in the closed, simply connected case with $4 \mid m$, as one readily sees by computing with Pontrjagin classes (the simplest example is the quaternion projective plane).

The classifying space $G / T O P$ has two distinct $H$-space structures, one given by the Whitney sum of bundles and one given by the disjoint union. The latter 
was originally defined by Sullivan using the characteristic variety theorem and by Quinn using the disjoint union of normal maps, and corresponds to the direct sum of quadratic Poincaré complexes in $\mathbb{L}_{0} \simeq G / T O P$. The abelian group structure on $[M, G / T O P]$ defined by + matches the abelian group structure on $H_{n}\left(M ; \mathbb{L}_{\bullet}\right)$. We shall now compare the composition formula for the structure invariant (Theorem 2.3) and the composition formula for the normal invariant (Proposition 2.7), using the expression of the Whitney sum $\oplus$ on $G / T O P$ as the addition

$$
\oplus: \mathbb{L}_{0} \times \mathbb{L}_{0} \rightarrow \mathbb{L}_{0} ;(a, b) \mapsto a \oplus b=a+b+a \otimes b
$$

(cf. Ranicki [16, p.295]).

Recall the definition of the Whitney sum of bundles $\eta: M \rightarrow B T O P(k)$, $\eta^{\prime}: M \rightarrow B T O P\left(k^{\prime}\right)$, namely as the composite

$\eta \oplus \eta^{\prime}: M \stackrel{\Delta}{\longrightarrow} M \times M \stackrel{\eta \times \eta^{\prime}}{\longrightarrow} B T O P(k) \times B T O P\left(k^{\prime}\right) \longrightarrow B T O P\left(k+k^{\prime}\right)$,

At this point, we need to recall the surgery product formula:

Proposition 3.1. (Ranicki [16, Proposition 8.1])

(i) The product $M \times M^{\prime}$ of an $n$-dimensional geometric Poincaré complex $M$ and an $n^{\prime}$-dimensional geometric Poincaré complex $M^{\prime}$ is an $\left(n+n^{\prime}\right)$-dimensional geometric Poincaré complex $M \times M^{\prime}$ with symmetric signature

$$
\begin{aligned}
& \sigma^{*}\left(M \times M^{\prime}\right)=\sigma^{*}(M) \otimes_{\mathbb{Z}} \sigma^{*}\left(M^{\prime}\right) \\
& \in L^{n+n^{\prime}}\left(\mathbb{Z}\left[\pi_{1}\left(M \times M^{\prime}\right)\right]\right)=L^{n+n^{\prime}}\left(\mathbb{Z}\left[\pi_{1}(M)\right] \otimes_{\mathbb{Z}} \mathbb{Z}\left[\pi_{1}\left(M^{\prime}\right)\right]\right) .
\end{aligned}
$$

(ii) The product of a normal map $(f, b): N \rightarrow M$ of n-dimensional geometric Poincaré complexes and a normal map $\left(f^{\prime}, b^{\prime}\right): N^{\prime} \rightarrow M^{\prime}$ of $n^{\prime}$-dimensional geometric Poincaré complexes is a normal map $\left(f \times f^{\prime}, b \times b^{\prime}\right): N \times N^{\prime} \rightarrow M \times M^{\prime}$ of $\left(n+n^{\prime}\right)$-dimensional geometric Poincaré complexes with quadratic signature

$$
\begin{gathered}
\sigma_{*}\left(f \times f^{\prime}, b \times b^{\prime}\right)=\sigma_{*}(f, b) \otimes \sigma^{*}\left(M^{\prime}\right)+\sigma^{*}(M) \otimes \sigma_{*}\left(f^{\prime}, b^{\prime}\right)+\sigma_{*}(f, b) \otimes \sigma_{*}\left(f^{\prime}, b^{\prime}\right) \\
\in L_{n+n^{\prime}}\left(\mathbb{Z}\left[\pi_{1}\left(M \times M^{\prime}\right)\right]\right)=L_{n+n^{\prime}}\left(\mathbb{Z}\left[\pi_{1}(M)\right] \otimes_{\mathbb{Z}} \mathbb{Z}\left[\pi_{1}\left(M^{\prime}\right)\right]\right) .
\end{gathered}
$$

Proof. (i) The symmetric complex of $M \times M^{\prime}$ is such that up homotopy equivalence

$$
\left(C\left(\widetilde{M \times M^{\prime}}\right), \phi_{M \times M^{\prime}}\right)=\left(C(\widetilde{M}), \phi_{M}\right) \otimes_{\mathbb{Z}}\left(C\left(\widetilde{M^{\prime}}\right), \phi_{M^{\prime}}\right)
$$


by the Eilenberg-Zilber theorem. Homotopy equivalent symmetric Poincaré complexes are cobordant.

(ii) The quadratic kernel of $\left(f \times f^{\prime}, b \times b^{\prime}\right)$ is such that up to homotopy equivalence

$$
\begin{aligned}
&\left(\mathcal{C}\left(\left(f \times f^{\prime}\right) !\right), \psi_{b \times b^{\prime}}\right)= \\
&\left(\left(\mathcal{C}\left(f^{!}\right), \psi_{b}\right) \otimes_{\mathbb{Z}}\left(C\left(\widetilde{M}^{\prime}\right), \phi_{M^{\prime}}\right)\right) \oplus\left(\left(C(\widetilde{M}), \phi_{M}\right) \otimes_{\mathbb{Z}}\left(\mathcal{C}\left(f^{\prime !}\right), \psi_{b^{\prime}}\right)\right) \\
& \oplus\left(\left(\mathcal{C}\left(f^{!}\right), \psi_{b}\right) \otimes_{\mathbb{Z}}\left(\mathcal{C}\left(f^{\prime !}\right), \psi_{b^{\prime}}\right)\right) .
\end{aligned}
$$

Since $\mathbb{L}^{\bullet}$ and $\mathbb{L} \bullet$ are ring spectra and $\mathbb{L} \bullet$ is an $\mathbb{L}^{\bullet}$-module spectrum there are defined cup and cap products for any space $M$

$$
\begin{aligned}
& \cup: H^{p}\left(M ; \mathbb{L}^{\bullet}\right) \otimes_{\mathbb{Z}} H^{q}\left(M ; \mathbb{L}^{\bullet}\right) \rightarrow H^{p+q}\left(M ; \mathbb{L}^{\bullet}\right), \\
& \cup: H^{p}\left(M ; \mathbb{L}_{\bullet}\right) \otimes_{\mathbb{Z}} H^{q}\left(M ; \mathbb{L}_{\bullet}\right) \rightarrow H^{p+q}\left(M ; \mathbb{L}_{\bullet}\right), \\
& \cap: H_{p}\left(M ; \mathbb{L}^{\bullet}\right) \otimes_{\mathbb{Z}} H^{q}\left(M ; \mathbb{L}_{\bullet}\right) \rightarrow H_{p-q}\left(M ; \mathbb{L}_{\bullet}\right) .
\end{aligned}
$$

The symmetric $L$-theory fundamental class $[M]_{\mathbb{L}} \in H_{n}\left(M ; \mathbb{L}^{\bullet}\right)$ of an $n$-dimensional manifold $M$ determines Poincaré duality isomorphisms

$$
[M]_{\mathbb{L}} \cap-: H^{*}\left(M ; \mathbb{L}_{\bullet}\right) \rightarrow H_{n-*}\left(M ; \mathbb{L}_{\bullet}\right) ; x \mapsto x_{*}=[M]_{\mathbb{L}} \cap x
$$

which can be used to define the intersection product

$$
H_{n-p}\left(M ; \mathbb{L}_{\bullet}\right) \otimes_{\mathbb{Z}} H_{n-q}\left(M ; \mathbb{L}_{\bullet}\right) \rightarrow H_{n-p-q}\left(M ; \mathbb{L}_{\bullet}\right) ; x_{*} \otimes y_{*} \mapsto x_{*} y_{*}=(x \cup y)_{*}
$$

and similarly

$$
H_{n-p}\left(M ; \mathbb{L}^{\bullet}\right) \otimes_{\mathbb{Z}} H_{n-q}\left(M ; \mathbb{L}_{\bullet}\right) \rightarrow H_{n-p-q}\left(M ; \mathbb{L}_{\bullet}\right) .
$$

We shall only be concerned with the intersection products in the special case $p=q=0$

$$
\begin{aligned}
& H_{n}\left(M ; \mathbb{L}_{\bullet}\right) \otimes_{\mathbb{Z}} H_{n}\left(M ; \mathbb{L}_{\bullet}\right) \rightarrow H_{n}\left(M ; \mathbb{L}_{\bullet}\right), \\
& H_{n}\left(M ; \mathbb{L}^{\bullet}\right) \otimes_{\mathbb{Z}} H_{n}\left(M ; \mathbb{L}_{\bullet}\right) \rightarrow H_{n}\left(M ; \mathbb{L}_{\bullet}\right) .
\end{aligned}
$$

Corollary 3.2. The product of an $n$-dimensional topological normal map $(f, b)$ : $N \rightarrow M$ and an $n^{\prime}$-dimensional topological normal map $\left(f^{\prime}, b^{\prime}\right): N^{\prime} \rightarrow M^{\prime}$ is an $\left(n+n^{\prime}\right)$-dimensional topological normal map

$$
\left(f \times f^{\prime}, b \times b^{\prime}\right): N \times N^{\prime} \rightarrow M \times M^{\prime}
$$

with topological normal invariant

$$
\eta\left(f \times f^{\prime}, b \times b^{\prime}\right)=\eta(f, b) \times \eta\left(f^{\prime}, b^{\prime}\right) \in\left[M \times M^{\prime}, G / T O P\right]
$$


and algebraic normal invariant

$$
\begin{gathered}
\begin{aligned}
t\left(f \times f^{\prime}, b \times b^{\prime}\right) & =t(f, b) \otimes\left[M^{\prime}\right]_{\mathbb{L}}+[M]_{\mathbb{L}} \otimes t\left(f^{\prime}, b^{\prime}\right)+t(f, b) \otimes t\left(f^{\prime}, b^{\prime}\right) \\
& =t(f, b) \otimes\left[M^{\prime}\right]_{\mathbb{L}}+f_{*}[N]_{\mathbb{L}} \otimes t\left(f^{\prime}, b^{\prime}\right) \in H_{n+n^{\prime}}\left(M \times M^{\prime} ; \mathbb{L}_{\bullet}\right)
\end{aligned} \\
\left(\text { using }(1+T) t(f, b)=f_{*}[N]_{\mathbb{L}}-[M]_{\mathbb{L}} \in H_{n}\left(M ; \mathbb{L}^{\bullet}\right)\right) .
\end{gathered}
$$

The Whitney sum of normal invariants has the following topological and algebraic properties:

Theorem 3.3. Let $(f, b): N \rightarrow M,\left(f^{\prime}, b^{\prime}\right): N^{\prime} \rightarrow M$ be normal maps of $n$ dimensional manifolds.

(i) The Whitney sum of the topological normal invariants $\eta(f, b), \eta\left(f^{\prime}, b^{\prime}\right) \in$ $[M, G / T O P]$ is the topological normal invariant

$$
\eta(f, b) \oplus \eta\left(f^{\prime}, b^{\prime}\right)=\eta\left(f^{\prime \prime}, b^{\prime \prime}\right)=\Delta^{*}\left(\eta(f, b) \times \eta\left(f^{\prime}, b^{\prime}\right)\right) \in[M, G / T O P]
$$

of the normal map $\left(f^{\prime \prime}, b^{\prime \prime}\right)$ obtained from the product

$$
\left(f \times f^{\prime}, b \times b^{\prime}\right): N \times N^{\prime} \rightarrow M \times M
$$

by transversality at the diagonal submanifold $\Delta: M \subset M \times M$

$$
\left(f^{\prime \prime}, b^{\prime \prime}\right)=\left(f \times f^{\prime}, b \times b^{\prime}\right) \mid: N^{\prime \prime}=\left(f \times f^{\prime}\right)^{-1}(M) \rightarrow M .
$$

(ii) The algebraic normal invariant of $\left(f^{\prime \prime}, b^{\prime \prime}\right): N^{\prime \prime} \rightarrow M$ is

$$
t\left(f^{\prime \prime}, b^{\prime \prime}\right)=t(f, b)+t\left(f^{\prime}, b^{\prime}\right)+t(f, b) t\left(f^{\prime}, b^{\prime}\right) \in H_{n}\left(M ; \mathbb{L}_{\bullet}\right)
$$

and surgery obstruction

$$
\begin{aligned}
\sigma_{*}\left(f^{\prime \prime}, b^{\prime \prime}\right) & =A\left(t\left(f^{\prime \prime}, b^{\prime \prime}\right)\right) \\
& =\sigma_{*}(f, b)+\sigma_{*}\left(f^{\prime}, b^{\prime}\right)+A\left(t(f, b) t\left(f^{\prime}, b^{\prime}\right)\right) \in L_{n}\left(\mathbb{Z}\left[\pi_{1}(M)\right]\right) .
\end{aligned}
$$

(iii) Suppose that

$$
\eta\left(f^{\prime}, b^{\prime}\right) \in \operatorname{im}\left(f^{*}:[N, G / T O P] \rightarrow[M, G / T O P]\right),
$$

so up to normal bordism there is a unique topological normal map $(g, c): P \rightarrow N$ with

$$
\begin{aligned}
& \eta(g, c)=f^{*} \eta\left(f^{\prime}, b^{\prime}\right) \in[N, G / T O P], \\
& t(g, c)=[N]_{\mathbb{L}} \cap \eta(g, c) \in H_{n}\left(N ; \mathbb{L}_{\bullet}\right), \\
& f_{*} t(g, c)=f_{*}[N]_{\mathbb{L}} \cap t\left(f^{\prime}, b^{\prime}\right)=t\left(f^{\prime}, b^{\prime}\right)+t(f, b) t\left(f^{\prime}, b^{\prime}\right) \in H_{n}\left(M ; \mathbb{L}_{\bullet}\right), \\
& \sigma_{*}(g, c)=A\left(f_{*} t(g, c)\right)=\sigma_{*}\left(f^{\prime}, b^{\prime}\right)+A\left(t(f, b) t\left(f^{\prime}, b^{\prime}\right)\right) \in L_{n}\left(\mathbb{Z}\left[\pi_{1}(M)\right]\right) .
\end{aligned}
$$


Then

$$
\eta\left(f^{\prime \prime}, b^{\prime \prime}\right)=\eta(f, b) \oplus \eta\left(f^{\prime}, b^{\prime}\right)=\eta(f g, b c) \in[M, G / T O P],
$$

so $\left(f^{\prime \prime}, b^{\prime \prime}\right): N^{\prime \prime} \rightarrow M$ is normal bordant to $(f g, b c): P \rightarrow M$, with

$$
\begin{aligned}
t\left(f^{\prime \prime}, b^{\prime \prime}\right) & =t(f, b)+t\left(f^{\prime}, b^{\prime}\right)+t(f, b) t\left(f^{\prime}, b^{\prime}\right) \\
& =t(f, b)+f_{*} t(g, c)=t(f g, b c) \in H_{n}\left(M ; \mathbb{L}_{\bullet}\right), \\
\sigma_{*}\left(f^{\prime \prime}, b^{\prime \prime}\right) & =\sigma_{*}(f, b)+\sigma_{*}\left(f^{\prime}, b^{\prime}\right)+A\left(t(f, b) t\left(f^{\prime}, b^{\prime}\right)\right) \\
& =\sigma_{*}(f, b)+f_{*} \sigma_{*}(g, c)=\sigma_{*}(f g, b c) \in L_{n}\left(\mathbb{Z}\left[\pi_{1}(M)\right]\right) .
\end{aligned}
$$

Proof. (i) By construction.

(ii) Pull back the product formulae of Corollary 3.2 along $\Delta: M \subset M \times M$, use $\nu_{\Delta}=\tau_{M}, \nu_{\Delta} \oplus \nu_{M} \simeq *$ and the commutative square

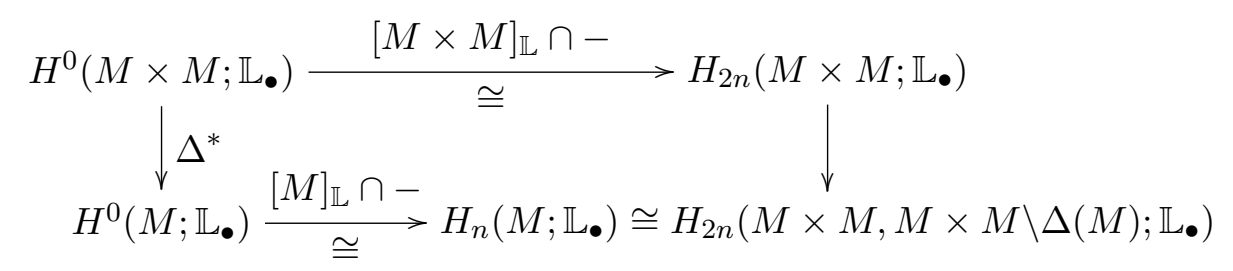

(iii) Immediate from (ii).

Remark 3.4. If $M, N, N^{\prime}$ are simplicial complexes and the normal maps $(f, b)$ : $N \rightarrow M,\left(f^{\prime}, b^{\prime}\right): N^{\prime} \rightarrow M$ in Theorem 3.3 are defined by simplicial maps $f: N \rightarrow M, f^{\prime}: N^{\prime} \rightarrow M$ to the barycentric subdivision of $M$ then the pullback $f^{\prime \prime}: N^{\prime \prime}=\left\{\left(x, x^{\prime}\right) \in N \times N^{\prime} \mid f(x)=f^{\prime}\left(x^{\prime}\right) \in M\right\} \rightarrow M ;\left(x, x^{\prime}\right) \mapsto f(x)=f^{\prime}\left(x^{\prime}\right)$ is a normal map $\left(f^{\prime \prime}, b^{\prime \prime}\right): N^{\prime \prime} \rightarrow M$ which is transverse at $\Delta: M \subset M \times M$, with kernel quadratic Poincaré complex in $\mathbb{A}(\mathbb{Z}, M)$ given up to homotopy equivalence by

$\left(\mathcal{C}\left(\left(f^{\prime \prime}\right)^{!}\right), \psi_{b^{\prime \prime}}\right)=\left(\mathcal{C}\left(f^{!}\right), \psi_{b}\right) \oplus\left(\mathcal{C}\left(f^{\prime !}\right), \psi_{b^{\prime}}\right) \oplus\left(\left(\mathcal{C}\left(f^{!}\right), \psi_{b}\right) \otimes_{\mathbb{A}(\mathbb{Z}, M)}\left(\mathcal{C}\left(f^{\prime !}\right), \psi_{b^{\prime}}\right)\right)$.

We now reconcile the manifold structure composition formula of Theorem 2.3 with the normal map composition formula (Proposition 2.7) of Brumfiel [1] and Madsen-Taylor-Williams [10]: 
Corollary 3.5. For homotopy equivalences of manifolds $f: N \rightarrow M, g: P \rightarrow N$ of $n$-dimensional manifolds

$$
\eta(N, f)+\eta\left(f_{*}(P, g)\right)=\eta(N, f) \oplus\left(f^{-1}\right)^{*} \eta(P, g) \in \mathcal{T}^{T O P}(M)=H_{n}\left(M ; \mathbb{L}_{\bullet}\right) .
$$

Proof. Let $(f, b): N \rightarrow M,(g, c): P \rightarrow N$ be the corresponding topological normal maps, with topological normal invariants

$$
\eta(N, f)=\eta(f, b) \in \mathcal{T}^{T O P}(M), \eta(P, g)=\eta(g, c) \in \mathcal{T}^{T O P}(N) .
$$

If $\left(f^{\prime}, b^{\prime}\right): N^{\prime} \rightarrow M$ is a topological normal map with

$$
\eta\left(f^{\prime}, b^{\prime}\right)=\left(f^{-1}\right)^{*} \eta(g, c) \in \mathcal{T}^{T O P}(M),
$$

then by Proposition 2.7 the Whitney sum topological normal map of Theorem 3.3 is

$$
\left(f^{\prime \prime}, b^{\prime \prime}\right)=(f g, b c): N^{\prime \prime}=P \rightarrow M
$$

up to normal bordism. Now

$$
\sigma_{*}(f, b)=\sigma_{*}(g, c)=\sigma_{*}(f g, b c)=0 \in L_{n}\left(\mathbb{Z}\left[\pi_{1}(M)\right]\right),
$$

and Theorem 3.3 (iii) gives

$$
\begin{aligned}
s(f g)= & s(f)+f_{*} s(g) \in \mathcal{S}_{n+1}(M), \\
t(f g, b c) & =t(f, b)+t\left(f^{\prime}, b^{\prime}\right)+t(f, b) t\left(f^{\prime}, b^{\prime}\right) \\
& =t(f, b)+f_{*} t(g, c) \in H_{n}\left(M ; \mathbb{L}_{\bullet}\right), \\
\sigma_{*}\left(f^{\prime}, b^{\prime}\right) & =-A\left(t(f, b) t\left(f^{\prime}, b^{\prime}\right)\right) \in L_{n}\left(\mathbb{Z}\left[\pi_{1}(M)\right]\right) .
\end{aligned}
$$

Let $h: Q \rightarrow M$ be a homotopy equivalence of $n$-dimensional manifolds with manifold structure $s(h)=f_{*} s(g) \in \mathcal{S}_{n+1}(M)$. The corresponding normal map $(h, d): Q \rightarrow M$ has algebraic normal invariant

$$
\begin{aligned}
& t(h, d)=f_{*} t(g, c)=t(f g, b c)-t(f, b)=t\left(f^{\prime}, b^{\prime}\right)+t(f, b) t\left(f^{\prime}, b^{\prime}\right) \\
& \quad \in \operatorname{im}\left(\mathcal{S}_{n+1}(M) \rightarrow H_{n}\left(M ; \mathbb{L}_{\bullet}\right)\right)=\operatorname{ker}\left(A: H_{n}\left(M ; \mathbb{L}_{\bullet}\right) \rightarrow L_{n}\left(\mathbb{Z}\left[\pi_{1}(M)\right]\right)\right) .
\end{aligned}
$$

The images of $(P, g) \in \mathcal{S}^{T O P}(N)$ in the noncommutative diagram

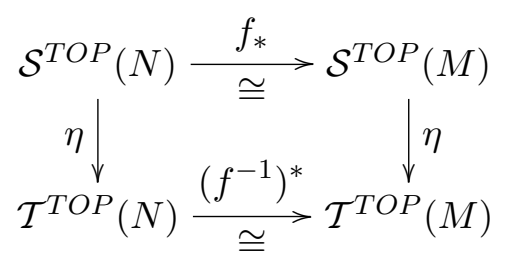


are given by

$$
\begin{aligned}
& \eta\left(f_{*}(P, g)\right)=\eta(Q, h)=t(h, d), \\
& \left(f^{-1}\right)^{*} \eta(P, g)=\left(f^{-1}\right)^{*} \eta(g, c)=t\left(f^{\prime}, b^{\prime}\right) \in \mathcal{T}^{T O P}(M)=H_{n}\left(M ; \mathbb{L}_{\bullet}\right),
\end{aligned}
$$

differing by

$$
t(h, d)-t\left(f^{\prime}, b^{\prime}\right)=t(f, b) t\left(f^{\prime}, b^{\prime}\right) \in \mathcal{T}^{T O P}(M)=H_{n}\left(M ; \mathbb{L}_{\bullet}\right) .
$$

Thus

$$
\begin{aligned}
\eta(N, f)+\eta\left(f_{*}(P, g)\right) & =t(f, b)+t(h, d) \\
& =t(f, b)+t\left(f^{\prime}, b^{\prime}\right)+t(f, b) t\left(f^{\prime}, b^{\prime}\right) \\
& =\eta(N, f) \oplus \eta\left(N^{\prime}, f^{\prime}\right) \\
& =\eta(N, f) \oplus\left(f^{-1}\right)^{*} \eta(P, g) \in \mathcal{T}^{T O P}(M)=H_{n}\left(M ; \mathbb{L}_{\bullet}\right) .
\end{aligned}
$$

We conclude with a specific example, $M=S^{p} \times S^{q}$, one of the two cases for which the manifold structure composition formula $s(f g)=s(f)+f_{*} s(g)$ of Theorem 2.3 is used by Kreck and Lück [8].

Example 3.6. (i) Let $M=S^{p} \times S^{q}$ for $p, q \geqslant 2$, so that $\pi_{1}(M)=\{1\}$. The assembly map in quadratic $L$-theory is given by

$$
A: H_{p+q}\left(M ; \mathbb{L}_{\bullet}\right)=L_{p}(\mathbb{Z}) \oplus L_{q}(\mathbb{Z}) \oplus L_{p+q}(\mathbb{Z}) \rightarrow L_{p+q}(\mathbb{Z}) ;(x, y, z) \mapsto z
$$

and

$$
\mathcal{S}_{p+q+1}(M)=\operatorname{ker}(A)=L_{p}(\mathbb{Z}) \oplus L_{q}(\mathbb{Z}) .
$$

The addition and intersection pairing in $H_{p+q}\left(M ; \mathbb{L}_{\bullet}\right)$ are given by

$$
\begin{aligned}
& (x, y, z)+\left(x^{\prime}, y^{\prime}, z^{\prime}\right)=\left(x+x^{\prime}, y+y^{\prime}, z+z^{\prime}\right) \\
& (x, y, z)\left(x^{\prime}, y^{\prime}, z^{\prime}\right)=\left(0,0, x y^{\prime}+x^{\prime} y\right) \in H_{p+q}\left(M ; \mathbb{L}_{\bullet}\right) .
\end{aligned}
$$

The product $L_{p}(\mathbb{Z}) \otimes L_{q}(\mathbb{Z}) \rightarrow L_{p+q}(\mathbb{Z})$ factors through $L_{p}(\mathbb{Z}) \otimes L^{q}(\mathbb{Z})$ and the quadratic and symmetric $L$-groups of $\mathbb{Z}$ are given by

$$
L_{n}(\mathbb{Z})=\left\{\begin{array}{l}
\mathbb{Z} \\
0 \\
\mathbb{Z}_{2} \\
0
\end{array} \quad, L^{n}(\mathbb{Z})=\left\{\begin{array} { l } 
{ \mathbb { Z } } \\
{ \mathbb { Z } _ { 2 } } \\
{ 0 } \\
{ 0 }
\end{array} \quad \text { for } n \equiv \left\{\begin{array}{l}
0 \\
1 \\
2 \\
3
\end{array} \quad(\bmod 4)\right.\right.\right.
$$


so the intersection pairing is non-zero only in the case $p \equiv q \equiv 0(\bmod 4)$. Given a topological normal map $(f, b): N \rightarrow M$ make $f$ transverse regular at $S^{p} \times\{*\}$, $\{*\} \times S^{q} \subset M$ to obtain topological normal maps

$$
\begin{aligned}
& \left(f_{p}, b_{p}\right)=(f, b) \mid: N_{p}=f^{-1}\left(S^{p} \times\{*\}\right) \rightarrow S^{p}, \\
& \left(f_{q}, b_{q}\right)=(f, b) \mid: N_{q}=f^{-1}\left(\{*\} \times S^{q}\right) \rightarrow S^{q} .
\end{aligned}
$$

and write the surgery obstructions as

$$
\left(\sigma_{*}\left(f_{p}, b_{p}\right), \sigma_{*}\left(f_{q}, b_{q}\right), \sigma_{*}(f, b)\right)=\left(x_{f}, y_{f}, z_{f}\right) \in L_{p}(\mathbb{Z}) \oplus L_{q}(\mathbb{Z}) \oplus L_{p+q}(\mathbb{Z}) .
$$

The algebraic normal invariant defines a bijection

$$
\mathcal{T}^{T O P}(M) \rightarrow L_{p}(\mathbb{Z}) \oplus L_{q}(\mathbb{Z}) \oplus L_{p+q}(\mathbb{Z}) ; \eta(f, b) \mapsto t(f, b)=\left(x_{f}, y_{f}, z_{f}\right) .
$$

The Whitney sum addition in $\mathcal{T}^{T O P}(M)$ corresponds to the addition

$$
(x, y, z) \oplus\left(x^{\prime}, y^{\prime}, z^{\prime}\right)=\left(x+x^{\prime}, y+y^{\prime}, x y^{\prime}+x^{\prime} y+z+z^{\prime}\right) \in L_{p}(\mathbb{Z}) \oplus L_{q}(\mathbb{Z}) \oplus L_{p+q}(\mathbb{Z}) .
$$

Given a homotopy equivalence $f: N \rightarrow M$ let $(f, b): N \rightarrow M$ be the corresponding topological normal map. The function

$$
\mathcal{S}^{T O P}(M) \rightarrow L_{p}(\mathbb{Z}) \oplus L_{q}(\mathbb{Z}) ; s(f) \mapsto\left(x_{f}, y_{f}\right)
$$

is a bijection, and

$$
t(f, b)=\left(x_{f}, y_{f}, 0\right) \in \mathcal{T}^{T O P}(M)=L_{p}(\mathbb{Z}) \oplus L_{q}(\mathbb{Z}) \oplus L_{p+q}(\mathbb{Z}) .
$$

Given also a homotopy equivalence $g: P \rightarrow N$ with corresponding topological normal map $(g, c): P \rightarrow N$ let

$$
f_{*} s(g)=\left(x_{g}, y_{g}\right) \in \mathcal{S}^{T O P}(M)=L_{p}(\mathbb{Z}) \oplus L_{q}(\mathbb{Z}),
$$

so that

$$
f_{*} t(g, c)=\left(x_{g}, y_{g}, 0\right) \in \mathcal{T}^{T O P}(M)=L_{p}(\mathbb{Z}) \oplus L_{q}(\mathbb{Z}) \oplus L_{p+q}(\mathbb{Z}) .
$$

As in the proof of Corollary 3.5 let $\left(f^{\prime}, b^{\prime}\right): N^{\prime} \rightarrow M$ be a topological normal map with topological normal invariant

$$
\eta\left(f^{\prime}, b^{\prime}\right)=\left(f^{-1}\right)^{*} \eta(g, c) \in \mathcal{T}^{T O P}(M),
$$

let $h: Q \rightarrow N$ be a homotopy equivalence with

$$
s(h)=f_{*} s(g)=\left(x_{g}, y_{g}\right) \in \mathcal{S}^{T O P}(M)=\mathcal{S}_{p+q+1}(M)=L_{p}(\mathbb{Z}) \oplus L_{q}(\mathbb{Z}),
$$


and let $(h, d): Q \rightarrow N$ be the corresponding topological normal map. Then

$$
\begin{aligned}
& \eta(f, b) \oplus \eta\left(f^{\prime}, b^{\prime}\right)=\eta(f g, b c) \in \mathcal{T}^{T O P}(M), \\
& \begin{aligned}
t\left(f^{\prime}, b^{\prime}\right)= & \left(x_{g}, y_{g},-x_{f} y_{g}-x_{g} y_{f}\right), t(h, d)=\left(x_{g}, y_{g}, 0\right), \\
t(f g, b c) & =t(f, b)+f_{*} t(g, c) \\
& =t(f, b) \oplus t\left(f^{\prime}, b^{\prime}\right)=t(f, b)+t\left(f^{\prime}, b^{\prime}\right)+t(f, b) t\left(f^{\prime}, b^{\prime}\right) \\
& =\left(x_{f}+x_{g}, y_{f}+y_{g}, 0\right) \in H_{p+q}\left(M ; \mathbb{L}_{\bullet}\right)=L_{p}(\mathbb{Z}) \oplus L_{q}(\mathbb{Z}) \oplus L_{p+q}(\mathbb{Z}), \\
s(f g)= & s(f)+f_{*} s(g)=\left(x_{f}+x_{g}, y_{f}+y_{g}\right) \in \mathcal{S}^{T O P}(M)=L_{p}(\mathbb{Z}) \oplus L_{q}(\mathbb{Z}) .
\end{aligned}
\end{aligned}
$$

(ii) For the simplest example of the non-additivity of the surgery obstruction function

$$
\theta: \mathcal{T}^{T O P}(M) \rightarrow L_{n}\left(\mathbb{Z}\left[\pi_{1}(M)\right]\right)
$$

with respect to $\oplus$ set $p=q=4$ in (i), and let $f: N \rightarrow M=S^{4} \times S^{4}$ be a homotopy equivalence with

$$
\begin{aligned}
& s(f)=(x, y) \in \mathcal{S}^{T O P}(M)=L_{4}(\mathbb{Z}) \oplus L_{4}(\mathbb{Z}), \\
& t(f, b)=(x, y, 0) \in \mathcal{T}^{T O P}(M)=L_{4}(\mathbb{Z}) \oplus L_{4}(\mathbb{Z}) \oplus L_{8}(\mathbb{Z}), \\
& \theta(\eta(f, b))=\sigma_{*}(f, b)=A(t(f, b))=0 \in L_{8}(\mathbb{Z})
\end{aligned}
$$

for any $x, y \in \mathbb{Z} \backslash\{0\}$. By (i) a homotopy inverse $g=f^{-1}: P=M \rightarrow N$ is then such that

$$
\begin{aligned}
& f_{*} s(g)=-s(f)=(-x,-y) \in \mathcal{S}^{T O P}(M)=L_{4}(\mathbb{Z}) \oplus L_{4}(\mathbb{Z}), \\
& f_{*} t(g, c)=-t(f, b)=(-x,-y, 0) \in \mathcal{T}^{T O P}(M)=L_{4}(\mathbb{Z}) \oplus L_{4}(\mathbb{Z}) \oplus L_{8}(\mathbb{Z})
\end{aligned}
$$

and a topological normal map $\left(f^{\prime}, b^{\prime}\right): N^{\prime} \rightarrow M$ with topological normal invariant $\eta\left(f^{\prime}, b^{\prime}\right)=\left(f^{-1}\right)^{*} \eta(g, c)$ has

$$
\begin{aligned}
& t\left(f^{\prime}, b^{\prime}\right)=(-x,-y, 2 x y) \in \mathcal{T}^{T O P}(M)=L_{4}(\mathbb{Z}) \oplus L_{4}(\mathbb{Z}) \oplus L_{8}(\mathbb{Z}), \\
& \theta\left(\eta\left(f^{\prime}, b^{\prime}\right)\right)=\sigma_{*}\left(f^{\prime}, b^{\prime}\right)=A\left(t\left(f^{\prime}, b^{\prime}\right)\right)=2 x y \neq 0 \in L_{8}(\mathbb{Z})=\mathbb{Z} .
\end{aligned}
$$

The Whitney sum

$$
\eta(f, b) \oplus \eta\left(f^{\prime}, b^{\prime}\right)=\eta(f g, b c)=\eta(1: M \rightarrow M)=0 \in \mathcal{T}^{T O P}(M)
$$

has surgery obstruction

$\sigma_{*}(f g, b c)=\sigma_{*}(f, b)+\sigma_{*}\left(f^{\prime}, b^{\prime}\right)+A\left(t(f, b) t\left(f^{\prime}, b^{\prime}\right)\right)=0+2 x y-2 x y=0 \in L_{8}(\mathbb{Z})$,

so

$$
\theta\left(\eta(f, b) \oplus \eta\left(f^{\prime}, b^{\prime}\right)\right)=0 \neq \theta(\eta(f, b))+\theta\left(\eta\left(f^{\prime}, b^{\prime}\right)\right)=2 x y \in L_{8}(\mathbb{Z})=\mathbb{Z} .
$$


Remark 3.7. See the preprint by Jahren and Kwasik [5] for an application of the composition formula obtained in this paper to the classification of free involutions on $S^{1} \times S^{n}$ for $n \geqslant 3$.

\section{REFERENCES}

[1] G.Brumfiel, Homotopy equivalences of almost smooth manifolds, Comm. Math. Helv. 46, 381-407 (1971)

[2] S.Cappell and S.Weinberger, A geometric interpretation of Siebenmann's periodicity phenomenon, Geometry and topology (Athens, Ga., 1985), Lecture Notes in Pure and Appl. Math. 105, 47-52, Dekker (1987)

[3] G.E.Cooke, The Hauptvermutung according to Casson and Sullivan (1968), published in The Hauptvermutung Book, 165-187, Kluwer (1996)

[4] M.Freedman and F.Quinn, Topology of 4-manifolds, Princeton Mathematical Series 39, Princeton University Press (1990)

[5] B.Jahren and S.Kwasik, Free involutions on $S^{1} \times S^{n}$, ArXiv e-print math.GT/08022035 (2008)

[6] L.Jones, Patch spaces: a geometric represntation for Poincaré spaces, Ann. of Maths. 97, 306-343 (1973), Corrections for patch spaces, ibid. 102, 183-185 (1975)

[7] R.Kirby and L.Siebenmann, Foundational Essays on Topological Manifolds, Smoothings, and Triangulations, Annals of Mathematics Studies 88, Princeton (1977)

[8] M.Kreck and W.Lück, Topological rigidity for non-aspherical manifolds, to appear in the Hirzebruch 80th birthday volume of the Quarterly Journal of Pure and Applied Mathematics, Part 2, e-print arXiv:math.GT/0509238

[9] T.Kro, Geometrically defined group structures in 3-dimensional surgery, Oslo Cand. Scient. Thesis (2000), http://www.maths.ntnu.no/toreak/hopp.pdf

[10] I.Madsen, L.Taylor and B.Williams, Tangential homotopy equivalences, Comm. Math. Helv. 55, 445-484 (1980)

[11] A.Nicas, Induction theorems for groups of homotopy manifold structures. Memoirs AMS $267(1982)$

[12] F.Quinn, A geometric formulation of surgery, Topology of manifolds, Proceedings 1969 Georgia Topology Conference, Markham Press, 500-511 (1970)

[13],$B_{\left(T O P_{n}\right)} \sim$ and the surgery obstruction. Bull. A.M.S. 77, 596-600 (1971)

[14] A.A.Ranicki, The total surgery obstruction, Proc. 1978 Arhus Topology Conference, Springer Lecture Notes in Mathematics 763, 275-316 (1979)

[15] _ The algebraic L-theory of surgery I. Foundations, Proc. L.M.S. (3) 40, 87-192 (1980)

[16] - The algebraic L-theory of surgery II. Applications to topology, Proc. L.M.S. (3) 40, 193-283 (1980) 
[17] _ Algebraic L-theory and topological manifolds, Tracts in Mathematics 102, Cambridge (1992)

[18] _ Singularities, double points, controlled topology and chain duality, Documenta Math. 4, 1-59 (1999)

[19] _ and M.Weiss, Chain complexes and assembly, Math. Z. 204, 157-185 (1990)

[20] L.Siebenmann, Approximating cellular maps by homeomorphisms, Topology 11, 271-294 (1972)

[21] C.T.C.Wall, Poincaré complexes, Ann. Math. 86, 213-245 (1967)

[22] _ _ Surgery on compact manifolds, Academic Press (1970), 2nd edition, A.M.S. (1999)

Andrew Ranicki

School of Mathematics and Maxwell Institute for Mathematical Sciences

University of Edinburgh

Edinburgh EH9 3JZ

Scotland, UK

E-mail: A.Ranicki@ed.ac.uk 